\title{
A clinical staging model for bipolar disorder: longitudinal approach
}

Lorena de la Fuente-Tomás ${ }^{1,2,3,4}$, Pilar Sierra ${ }^{5}$, Mónica Sanchez-Autet @ ${ }^{6}$, Belén Arranz ${ }^{1,6}$, Ana García-Blanco ${ }^{5}$, Gemma Safont ${ }^{1,7}$ and Maria P. García-Portilla ${ }^{1,2,3,4,8}$

\begin{abstract}
Bipolar disorder (BD) has been identified as a life-course illness with different clinical manifestations from an at-risk to a late stage, supporting the assumption that it would benefit from a staging model. In a previous study, we used a clustering approach to stratify 224 patients with a diagnosis of BD into five clusters based on clinical characteristics, functioning, cognition, general health, and health-related quality of life. This study was design to test the construct validity of our previously developed $k$-means clustering model and to confirm its longitudinal validity over a span of 3 years. Of the 224 patients included at baseline who were used to develop our model, 129 (57.6\%) reached the 3-year follow-up. All life domains except mental health-related quality of life (QoL) showed significant worsening in stages $(p<0.001)$, suggesting construct validity. Furthermore, as patients progressed through stages, functional decline $(p<$ $0.001)$ and more complex treatment patterns $(p=0.002)$ were observed. As expected, at 3 years, the majority of patients remained at the same stage $(49.6 \%)$, or progressed (20.9\%) or regressed $(23.3 \%)$ one stage. Furthermore, $85 \%$ of patients who stayed euthymic during that period remained at the same stage or regressed to previous stages, supporting its longitudinal validity. For that reason, this study provides evidence of the construct and longitudinal validity of an empirically developed, comprehensive staging model for patients with BD. Thus, it may help clinicians and researchers to better understand the disorder and, at the same time, to design more accurate and personalized treatment plans.
\end{abstract}

\section{Introduction}

Bipolar disorder (BD) is a life-course illness characterized by alternation of periods of euthymia with depressive, manic, and mixed episodes. Recently, it has been reconceptualized as a changing disorder with different clinical manifestations over the course of its development from an at-risk or latent stage to a late or end-stage, thus supporting the assumption that it would benefit from a staging model $^{1}$. Clinical manifestations consistent with staging models include cognitive deterioration and functional decline $e^{2,3}$, changes in inflammatory and

Correspondence: Lorena de la Fuente-Tomás (lorenadelafuentetomas@gmail. com)

${ }^{1}$ Centro de Investigación Biomédica en Red de Salud Mental (CIBERSAM),

Madrid, Spain

${ }^{2}$ Department of Psychiatry, University of Oviedo, Oviedo, Spain

Full list of author information is available at the end of the article. neuroanatomical biomarkers ${ }^{3-9}$, less response to treatment $^{10}$, and worse self-reported quality of life $(\mathrm{QoL})^{11}$ linked to disorder progression.

Although different staging models have been proposed from a theoretical perspective ${ }^{12-16}$ studies on BD with an empirical staging-development and longitudinal-data approach are scarce ${ }^{17}$. So far, only one study ${ }^{18}$ has tested the applicability of a theoretical clinical staging model for BD progressively developed by different authors ${ }^{12-14}$.

Recent research showed that BD could be fit to a mathematical model ${ }^{19,20}$. In a previous study, we developed a comprehensive, evidence-based $k$-means clustering model for BD that distinguishes five clusters ranging from the least severe (stage 1) to the most severe one (stage 5) based on clinical characteristics, physical health, cognition performance, real world functioning, and health-related QoL ${ }^{21}$. Now, with this study, we aim to use

\section{(c) The Author(s) 2020}

(c) (i) Open Access This article is licensed under a Creative Commons Attribution 4.0 International License, which permits use, sharing, adaptation, distribution and reproduction c. in any medium or format, as long as you give appropriate credit to the original author(s) and the source, provide a link to the Creative Commons license, and indicate if changes were made. The images or other third party material in this article are included in the article's Creative Commons license, unless indicated otherwise in a credit line to the material. If material is not included in the article's Creative Commons license and your intended use is not permitted by statutory regulation or exceeds the permitted use, you will need to obtain permission directly from the copyright holder. To view a copy of this license, visit http://creativecommons.org/licenses/by/4.0/. 
a different sample to test the construct validity of our model as well as its longitudinal validity, thereby providing proof of its validity as a staging model for use in patients with BD. We hypothesized that our model would behave properly with a different sample and that, at 3-year follow-up, the majority of the patients would remain at the same stage or would progress or regress one stage, while only a small proportion of patients would progress or regress two or more stages.

\section{Materials and methods}

This is a prospective, 3-year follow-up, multicenter study conducted at four sites in Spain (Oviedo, Barcelona, and Valencia) with the aim to develop and validate an empirical staging model for using in patients with BD.

The baseline study was conducted between April 2012 and December 2014 (ref. PI11/02493), and the 3-year follow-up was conducted between April 2015 and July 2018 (ref. PI14/02037). The Clinical Research Ethics Committee of Hospital Universitario Central de Asturias in Oviedo approved the study protocol (refs. 36/12 and 142/15). Written informed consent was obtained from all participants prior to enrollment.

\section{Participants}

Of the 224 patients enrolled at baseline, 129 (57.6\%) completed the 3-year follow-up assessment. Inclusion criteria at baseline were: (1) outpatients with a SCID-Iconfirmed diagnosis of BD according to DSM-IV-TR ${ }^{22}$ in treatment at any of the four participating sites; (2) age $\geq$ 18 years; and (3) written informed consent to participate in the study. Exclusion criteria consisted only of refusal to participate in the study.

\section{Assessments}

Assessments were identical at baseline and at 3-year follow-up and included: (1) demographic and clinical information obtained from the clinical records of the patients (clinical course and specific characteristics of BD, psychiatric and physical comorbidities, officially recognized disability, and psychopharmacological treatments); (2) psychometric assessment: (2a) clinician-rated outcome measures (CROMs): Spanish versions of Hamilton Depression Rating Scale (HDRS) ${ }^{23}$, Hamilton Anxiety Rating Scale $(\mathrm{HARS})^{24}$, Young Mania Rating Scale $(\text { YMRS })^{25}$, Clinical Global Impression $(\mathrm{CGI})^{26}$, Oviedo Sleep Questionnaire (OSQ $)^{27}$, Changes in Sexual Functioning Questionnaire (CSFQ) ${ }^{28}$, Scale for Cognitive Impairment in Psychiatry (SCIP) ${ }^{29}$, Global Assessment of Functioning $(\mathrm{GAF})^{30}$, and Functioning Assessment Short Test $(\mathrm{FAST})^{31}$; (2b) Patient-Reported Outcome Measures (PROMs): the Spanish version of MOS 36-item ShortForm Health-Survey $(\mathrm{SF}-36)^{32}$; (3) anthropometry [height, weight, waist circumference, and body mass index], vital signs (heart rate and blood pressure), and lab results [hematology (erythrocytes, hemoglobin, leukocytes, platelets), lipid profile (cholesterol, LDL cholesterol, HDL cholesterol, triglycerides), glucose, hepatic function (GPT, GOT, GGT, bilirubin), renal function (creatinine, BUN), hormones (PRL, TSH), and inflammatory and oxidative biomarkers (CRP, homocysteine)] were collected (for further detail, see Fuente-Tomas et al. ${ }^{21}$ ).

\section{Our staging model}

The first step in the development of our staging model was to create a cluster-based method to classify patients with $\mathrm{BD}$ using a cross-sectional sample ${ }^{21}$. We made a dimensional reduction using $k$-means clustering. This technique aims to partition $n$ observations into $k$ clusters in which each observation belongs to the cluster with the nearest mean. Comparisons of between-group variables were then performed by Chi-square and univariate ANOVA followed by Tukey's honestly significant difference post-hoc testing. Those variables in which statistically significant differences between groups were found were selected to be part of the model along with other variables added by expert criteria. We used all these variables, hereafter called profilers, to calculate a global severity formula.

Using the severity formula shown below, we obtain a global severity score for each patient which allows us to assign that patient to one of the five clusters of the staging model.

$$
\begin{aligned}
\text { Severity }= & \frac{10}{12} \cdot\left(\mathrm{PD} x \mathrm{BD}+\mathrm{MetS}+\mathrm{ComPD}+\mathrm{SCIP}_{\mathrm{T}_{r 4}}\right. \\
& + \text { IllnessN } \mathrm{SFPF}+\mathrm{SFMH}+\mathrm{FAST}_{\mathrm{T}} \\
& \left.+ \text { FAST }_{\text {leisure }}+\mathrm{BMI}+\text { HospN }+ \text { SuicAttN }\right)
\end{aligned}
$$

The formula includes 12 profilers from the following five life domains: (1) Clinical characteristics of the BD: three profilers: Number of hospitalizations (HospN), Number of suicide attempts (SuicAttN), and Comorbid personality disorder (ComPD); (2) Physical health: three profilers: Body Mass Index (BMI), Metabolic Syndrome (MetS), and Number of comorbid physical illnesses (IllnessN); (3) Cognition: one profiler: Screen for Cognitive Impairment in Psychiatry score $\left(\mathrm{SCIP}_{\mathrm{Tr} 4}\right)$; (4) real-world functioning: three profilers: permanently disabled due to $\mathrm{BD}(\mathrm{PD} \times \mathrm{BD})$, Functioning Assessment Short Test total score $\left(\mathrm{FAST}_{\mathrm{T}}\right)$, and Functioning Assessment Short Test leisure time subscale score (FAST leisure $_{\text {e }}$; and (5) Healthrelated QoL: two profilers: SF-36 Physical Functioning Scale score (SFPF), and SF-36 Mental Health Scale score (SF-MH). All profilers have the same weight and may take values between 0 and 1 , so the severity score ranges from 0 to 10. Based on this score, we proposed the cut-off for delimiting the five clusters using the scores corresponding 
to the 5th, 25th, 50th, 75th, and 95th percentiles (1.70, 2.50 , 4.50, and 6.10 , and $\geq 6.11$, respectively).

The second step, described in this paper, was to further validate our classification model as regards construct validity and longitudinal validity with the original sample at 3-year follow-up.

\section{Statistical analysis}

Analyses were conducted using IBM SPSS Statistics for Windows, Version 22.0. The significance level was set at $p<0.05$. We used a chi-squared test, paired $t$-test, and ANOVA with Tukey post-hoc test to identify associations between variables.

We tested for construct validity of our staging model by examining if: (1) all the profilers included in the model behave properly, that is, if patients get more severe scores on each profiler in late stages than in early stages and (2) our proposed external validators (GAF scores and pharmacological treatment patterns) also behave properly. We hypothesized that, in late stages, the global level of functioning would be more impaired and the prescribed pharmacological treatment more complex.

Concerning longitudinal validity, we analyzed the shift of patients throughout the model from baseline to 3-year follow-up. Here, we expected patients to move slightly forward or backward along the model with a very small percentage presenting greater changes (more than two stages). Furthermore, we expected a large proportion (more than $50 \%$ ) of patients who stayed euthymic during the 3-year follow-up period to remain in the same stage.

\section{Results}

On average, the mean follow-up time was 37.9 ( $\mathrm{SD}=$ 2.1) months. At 3-year follow-up, 129 (57.6\%) patients were reassessed.

\section{Demographic and clinical characteristics}

Table 1 shows participant demographic and clinical characteristics, including the profilers of the model. Patients had a mean age of $50.3(\mathrm{SD}=12.0)$, and the majority were female (65.2\%) and Caucasian (96.2\%). Diagnoses were as follows: 73\% had BD I, 23 (17.4\%) a comorbid personality disorder, and $9(7 \%)$ a substance use disorder. Furthermore, 38 (32.2\%) patients remained in a euthymic state throughout the follow-up period.

The mean CGI-S score was $3.27(\mathrm{SD}=1.4)$. Regarding psychopathology, $62(47 \%)$ had a score consistent with bipolar depression according to the HDRS $(\geq 7)^{33}, 12$ (9.1\%) with a mixed episode (YMRS $\geq 7-20)$ and 4 (3\%) with a manic episode according to the YMRS $(>20)$. Concerning the cognitive assessment, 25 (19.4\%) had mild, 27 (20.9\%) moderate, and 26 (20.2\%) severe impairment.
On average, patients were receiving $3.2(\mathrm{SD}=1.4)$ prescribed drugs. One hundred seventeen (90.7\%) patients were taking one classic mood stabilizer, 29 (22.5\%) were taking a combination of two, $78(60.5 \%)$ at least one antipsychotic, 19 (14.7\%) a combination of two, 51 (39.5\%) antidepressants, and 65 (49.6\%) benzodiazepines.

\section{Classification of the patients in the staging model}

Of the 129 patients followed at 3 years, 14 (10.9\%) were classified as stage 1, $20(15.5 \%)$ as stage 2, $61(47.3 \%)$ as stage 3 , and $24(18.6 \%)$ as stage 4 , and $10(7.8 \%)$ as stage 5 . Their mean global severity score was $3.6(\mathrm{SD}=1.6)$, with a minimum of 0.9 and a maximum of 8.4. At baseline, their mean global severity score was $3.6(\mathrm{SD}=1.4)$, with a minimum value of 0.8 and maximum of 8.0 (see Fig. 1). The mean global severity score of patients who did not complete follow-up was $3.5(\mathrm{SD}=1.3)$. We did not find significant differences in age, gender, bipolar type, age at onset, and total FAST score between followed and lost patients.

\section{Construct validity}

As can be seen in Table 2, except for SF-36 mental health, all profilers became significantly worse as they progressed through the stages, thus providing proof of construct validity. Furthermore, evidence of construct validity was also provided by the external validators. Concerning GAF scores, significant worsening was seen as the stages progressed, ranging from $81.1(\mathrm{SD}=11.9)$ in stage 1 to $49.5(\mathrm{SD}=13.4)$ in stage 2 (see Table 3). Finally, regarding pharmacological treatment patterns, early stages ( 1 and 2 ) were associated with monotherapy or use of two-drug combinations, while late stages (4 and 5) were associated with combinations of four or more drugs $(p=0.002)$. Also, patients in late stages more frequently received antidepressants and benzodiazepines (see Table 3).

\section{Longitudinal validity}

Figure 2 shows the shift of patients throughout the model at 3-year follow-up. Specifically, $50 \%$ of patients at stage 1 progressed to stage 2 and $16.7 \%$ to stage 3 . Regarding stage 2, 27.6\% of patients regressed one stage, while $37.9 \%$ progressed to stage 3 and only one (3.4\%) advanced to stage 4 . The majority of those at stage 3 remained at that stage $(63.3 \%)$, while $18.2 \%$ regressed or progressed one stage. Regarding stage $4,32 \%$ regressed to stage 3 and $26.3 \%$ progressed to stage 5 . Finally, one-third of patients at stage 5 remained at that stage, while $55.6 \%$ regressed to stage 4 and $1(11.1 \%)$ to stage 3.

When looking at the shifts in patients who stayed euthymic during the 3-year follow-up period, almost all remained at the same stage $(55.3 \%)$ or regressed $(23.7 \%)$ 
Table 1 Patient demographic and clinical characteristics.

\begin{tabular}{|c|c|c|}
\hline Sample characteristics & Follow-up mean (SD) $(n=129)$ & Baseline mean (SD) $(n=129)$ \\
\hline Mean age [mean (SD)] & $50.3(12.0)$ & $46.9(12.0)$ \\
\hline Sex, females $[n(\%)]$ & $86(65.2)$ & $86(65.2)$ \\
\hline Length of illness, years [mean (SD)] & $23.6(12.4)$ & $20.5(12.3)$ \\
\hline Global assessment of functioning (GAF) [mean (SD)] & $68.2(14.0)$ & $67.4(15.5)$ \\
\hline Suicide attempts ${ }^{\mathrm{a}}$, yes $[n(\%)]$ & $9(6.8)$ & NA \\
\hline Hospitalizations ${ }^{\mathrm{a}}$, yes $[n(\%)]$ & $23(17.4)$ & NA \\
\hline Manic episodes ${ }^{a}$, yes $[n(\%)]$ & $25(19.8)$ & NA \\
\hline Depressive episodes ${ }^{a}$, yes $[n(\%)]$ & $43(33.9)$ & NA \\
\hline \multicolumn{3}{|l|}{ Profilers (direct scores) } \\
\hline Number of hospitalizations [mean (SD)] & $1.2(2.0)$ & $2.3(2.8)$ \\
\hline Number of suicide attempts [mean (SD)] & $1.0(2.0)$ & $0.9(1.9)$ \\
\hline Comorbid personality disorder, yes $[n(\%)]$ & $23(17.4)$ & $24(18.6)$ \\
\hline Body mass index [mean (SD)] & $24.5(4.9)$ & $28.7(5.6)$ \\
\hline Metabolic syndrome, yes $[n(\%)]$ & $52(39.4)$ & $34(27.6)$ \\
\hline Number of comorbid physical illnesses [mean (SD)] & $1.7(1.6)$ & $1.9(1.2)$ \\
\hline SCIP category, no cognitive impairment $[n(\%)]$ & $51(39.8)$ & $34(27.0)$ \\
\hline Permanent disability due to bipolar disorder, yes [n (\%)] & $63(47.8)$ & $59(45.7)$ \\
\hline FAST total score [mean (SD)] & $26.8(15.8)$ & $26.6(16.8)$ \\
\hline FAST leisure subscale score [mean (SD)] & $2.2(2.1)$ & $3.0(7.1)$ \\
\hline SF-36 physical functioning scale score [mean (SD)] & $-0.5(0.7)$ & $-0.5(0.6)$ \\
\hline SF-36 mental health scale score [mean (SD)] & $-1.1(2.1)$ & $-1.1(1.8)$ \\
\hline \multicolumn{3}{|l|}{ Profilers (transformed score $0-1$ ) } \\
\hline Number of hospitalizations [mean (SD)] & $0.19(0.3)$ & $0.37(0.3)$ \\
\hline Number of suicide attempts [mean (SD)] & $0.18(0.3)$ & $0.15(0.3)$ \\
\hline Comorbid personality disorder, yes $[n(\%)]$ & $0.18(0.4)$ & $0.18(0.4)$ \\
\hline Body mass index [mean (SD)] & $0.15(0.2)$ & $0.32(0.3)$ \\
\hline Metabolic syndrome, yes [n (\%)] & $0.39(0.5)$ & $0.34(0.5)$ \\
\hline Number of comorbid physical illnesses [mean (SD)] & $0.39(0.3)$ & $0.26(0.3)$ \\
\hline SCIP category, no cognitive impairment $[n(\%)]$ & $0.47(0.4)$ & $0.40(0.3)$ \\
\hline Permanent disability due to bipolar disorder, yes $[n(\%)]$ & $0.47(0.5)$ & $0.46(0.5)$ \\
\hline FAST total score [mean (SD)] & $0.37(0.2)$ & $0.37(0.2)$ \\
\hline FAST leisure subscale score [mean (SD)] & $0.33(0.3)$ & $0.40(0.3)$ \\
\hline SF-36 physical functioning scale score [mean (SD)] & $0.53(0.1)$ & $0.55(0.2)$ \\
\hline SF-36 mental health scale score [mean (SD)] & $0.62(0.2)$ & $0.55(0.1)$ \\
\hline
\end{tabular}

${ }^{\text {a }}$ During the 3-year follow-up period.

or advanced (15.8\%) one stage. Two patients (5.3\%) regressed two stages. In those patients who remained at the same stage or regressed to previous ones, there were statistically significant improvements in the clinical $(t=3.732, p=0.001$ and $t=5.090, p<0.001$, respectively), functioning ( $t=2.626, p=0.016$ and $t=3.705$, $p=0.004$, respectively), and QoL dimensions $(t=8.000$, $p<0.001$ and $t=3.184, p=0.010$, respectively).

\section{Discussion}

Our results demonstrate that our staging model has good construct and longitudinal validity, thus supporting its use 


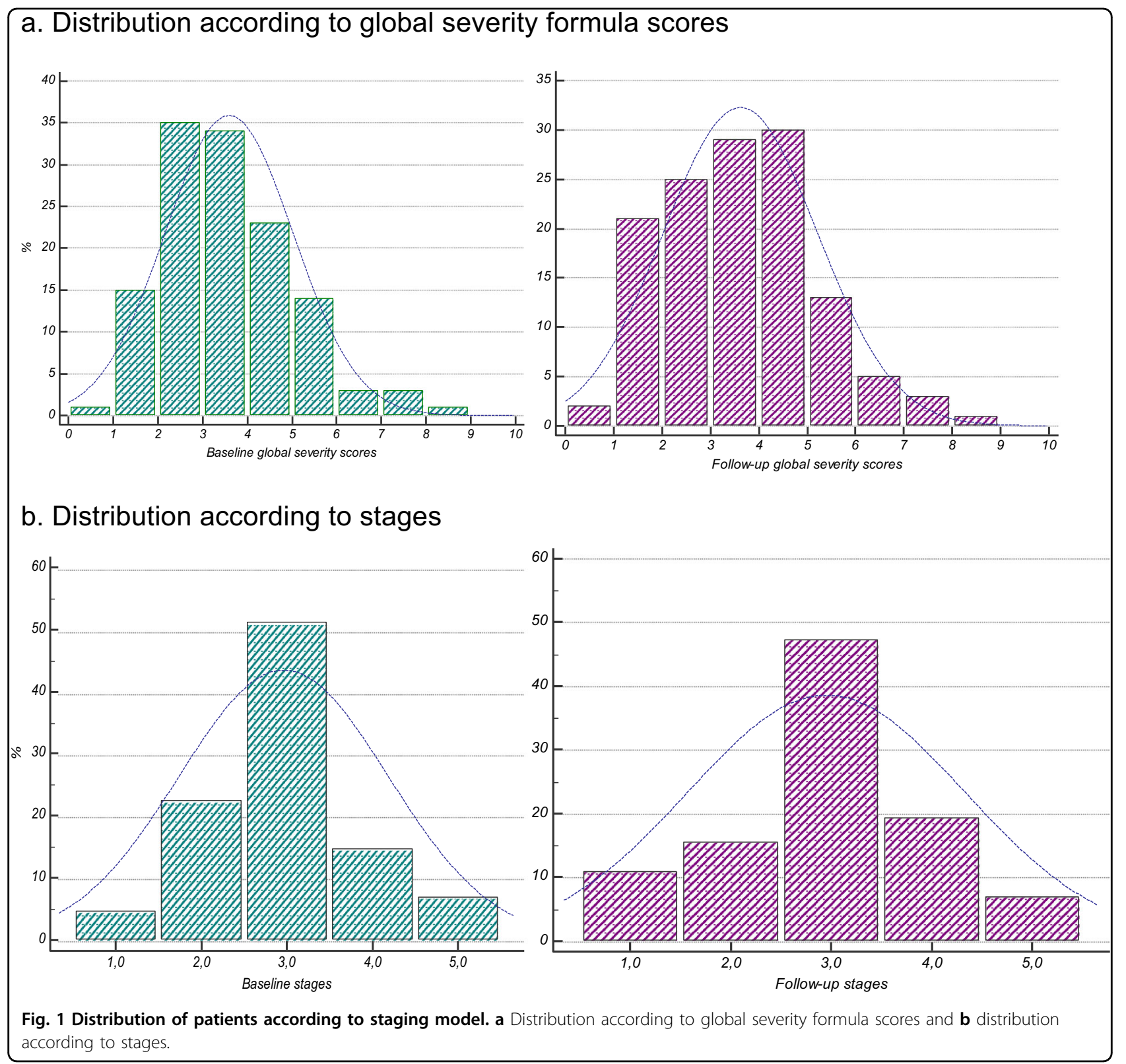

in daily clinical practice. Regarding construct validity, with the exception of the mental health-related QoL profiler, all behave properly, showing significant worsening through the stages. Furthermore, the proposed external validators (GAF scores and pharmacological treatment pattern) also behave properly, that is, there is a functional decline across stages, and pharmacological treatment patterns are more complex at late stages than at early ones. Concerning longitudinal validity, at 3-year follow-up, the shift of patients throughout the model was as expected considering the short follow-up period, with half remaining at the same stage, $40 \%$ progressing or regressing one stage, and fewer than $10 \%$ progressing or regressing two.
Notwithstanding the fact that the course of BD is heterogeneous, there is evidence for clinical progression ${ }^{34}$, and accordingly, the five life domains of our staging model showed this progression. Regarding the clinical characteristics of $\mathrm{BD}$, patients at late stages experienced more hospitalizations and suicide attempts and more frequently had a comorbid personality disorder. Consistent with these data, one study reported the same results between patients with first and multiple mood episodes ${ }^{35}$. However, two other studies ${ }^{2,36}$ did not find this clinical pattern among patients in different stages. This discrepancy may be due to the criteria used to classify patients into stages. In both of those studies, patients were assigned to the 


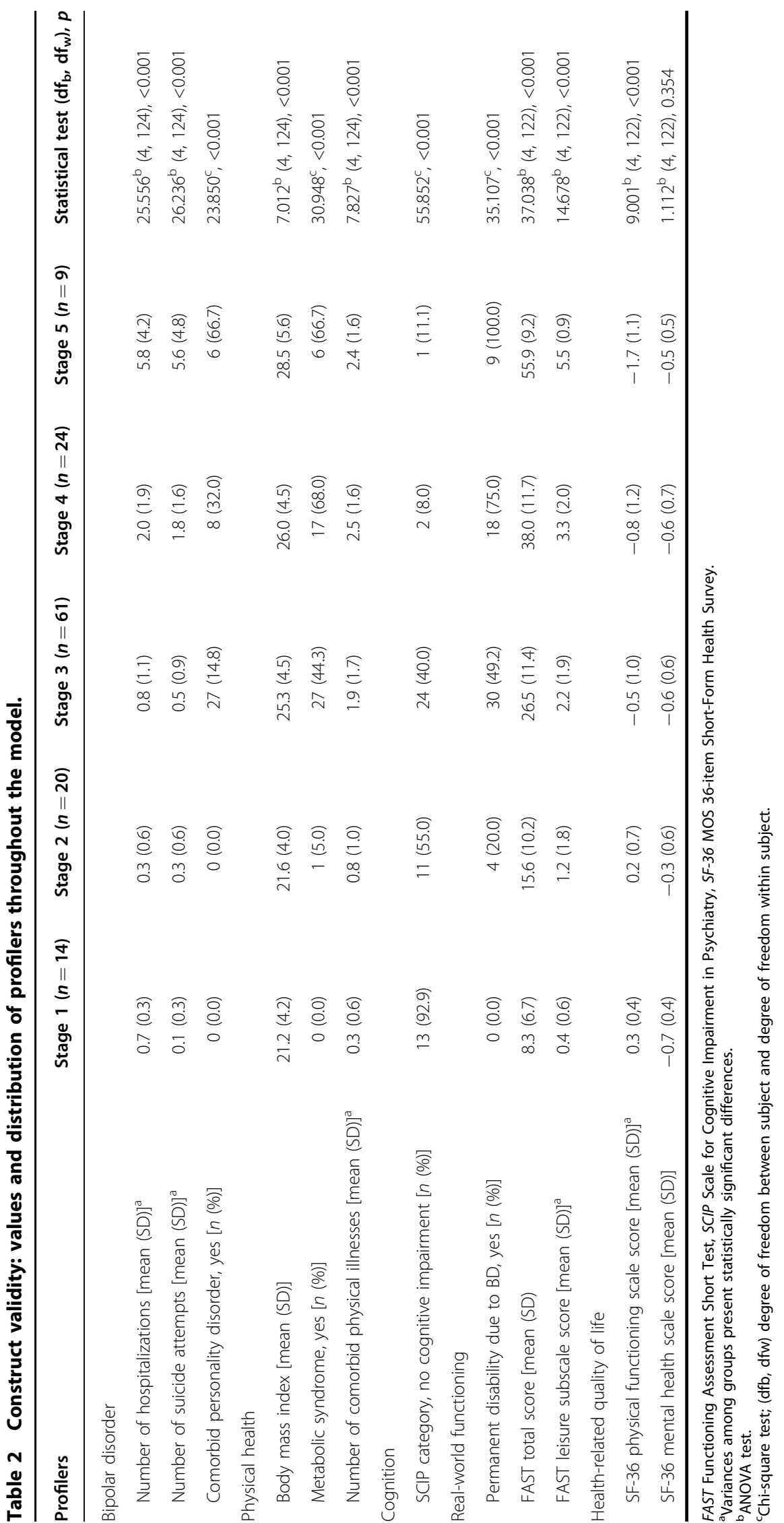


Table 3 Construct validity, external validators: GAF scores and pharmacological treatment patterns throughout the model.

\begin{tabular}{|c|c|c|c|c|c|c|}
\hline & Stage $1(n=14)$ & Stage $2(n=20)$ & Stage $3(n=61)$ & Stage $4(n=25)$ & Stage $5(n=9)$ & Statistical test $\left(\mathrm{df}_{\mathrm{b}}, \mathrm{df}_{\mathrm{w}}\right), p$ \\
\hline GAF scores [mean (SD)] & $81.1(11.9)$ & $79.5(9.2)$ & $67.6(11.4)$ & $62.3(10.8)$ & $47.2(12.0)$ & $20.385^{a}(4,123),<0.001$ \\
\hline Number of prescribed drugs [n(\%)] & & & & & & $43.257^{b}, 0.002$ \\
\hline One drug & $4(30.8)$ & $5(26.3)$ & $2(3.7)$ & $1(4.5)$ & $0(0.0)$ & \\
\hline Two drugs & $5(38.5)$ & $8(42.1)$ & $14(25.9)$ & $3(13.6)$ & $0(0.0)$ & \\
\hline Three drugs & $1(7.7)$ & $4(21.1)$ & $15(27.8)$ & $7(31.8)$ & $0(0.0)$ & \\
\hline Four drugs & $2(15.4)$ & $1(5.3)$ & $12(22.2)$ & $5(22.7)$ & $5(55.6)$ & \\
\hline Five or more drugs & $1(7.7)$ & $1(5.3)$ & $7(13.0)$ & $4(18.2)$ & $4(44.4)$ & \\
\hline \multicolumn{7}{|l|}{ Type of prescribed drugs [n (\%)] } \\
\hline Mood stabilizers & & & & & & $1.957^{b}, 0.744$ \\
\hline One drug & $8(66.7)$ & $15(83.3)$ & $41(80.4)$ & $6(28.6)$ & $2(28.6)$ & \\
\hline Two drugs & $4(33.3)$ & $3(16.7)$ & $10(19.6)$ & $9(19.1)$ & $7(53.8)$ & \\
\hline Antipsychotics & $7(50.0)$ & $8(40.0)$ & $37(60.7)$ & $16(66.7)$ & $8(80.8)$ & $5.925^{b}, 0.205$ \\
\hline Antidepressants & $3(21.4)$ & $6(30.0)$ & $31(51.7)$ & $11(45.8)$ & $9(90.0)$ & $13.960^{\mathrm{b}}, 0.007$ \\
\hline Benzodiazepines & $3(21.4)$ & $6(31.6)$ & $31(50.8)$ & $16(66.7)$ & $8(80.0)$ & $13.430^{b}, 0.009$ \\
\hline
\end{tabular}

GAF global assessment of functioning.

${ }^{a}$ ANOVA test.

${ }^{b}$ Chi-square test; (dfb, dfw) degree of freedom between subject and degree of freedom within subject.

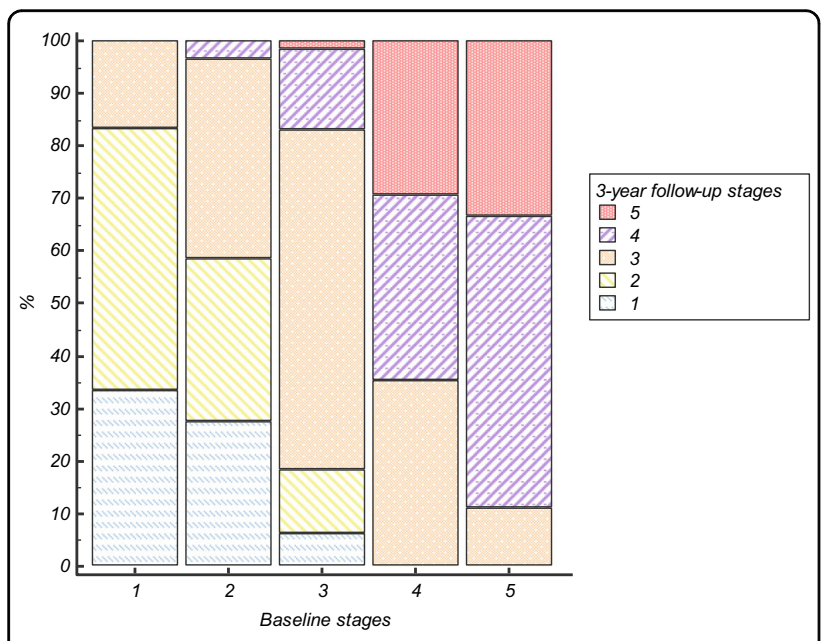

Fig. 2 Shift throughout the model at 3 years of follow-up.

different stages based on functional impairment only, and not multiple domains of life. In addition, some of our profilers in this dimension were not used in those studies. Physical health, cognition, and functioning were the domains in which patients showed the most remarkable progressive worsening, thus identifying a score-dependent pattern. These findings comport with previous reports showing cognitive and functional decline along with the progression of $\mathrm{BD}^{2,36,37}$ and with theoretical models proposed by Kapczinski et al. (2009) ${ }^{12}$ and Cosci and Fava $(2013)^{38}$. Concerning self-reported QoL, as the disorder progresses, physical QoL seems to worsen. In agreement with our results, a recent study by Tatay-Manteiga et al. $(2019)^{11}$ showed that BD patients reported poorer QoL in late than early stages in physical, psychological, social, and environment domains. However, again, that study used a different criterion to classify patients based solely on FAST scores.

We have identified only one study that examined a staging model for BD from a longitudinal perspective ${ }^{18}$, although its aim was to find the patient characteristics that define their progression throughout the model. In our study, over the 3 years of follow-up, patients shifted across stages as expected, that is, in that very short period of time, only five patients had strong shifts (progressing or regressing two or more stages). Unfortunately, we did not find standardized patterns for transition over time in BD staging models to contrast with our results. Further proof of the longitudinal validity of our model is that most patients who were in a euthymic state remained at the same stage or had regressed to a previous one at followup. Although functional ${ }^{39}$ and cognitive ${ }^{40}$ impairments have been associated with subsyndromal depressive symptoms in cross-sectional studies, our longitudinal results demonstrate a statistically significant improvement in functioning and QoL dimensions in those patients who remained euthymic for 36 months and who remained at the same stage or regressed to a previous stage, thus calling into question the association reported in the literature.

All these findings support the construct and longitudinal validity of our model for patients with $\mathrm{BD}^{21}$ and provide further support for using this clinical staging model in clinical practice, taking into account the easy access to profiles in any clinical environment. We would like to highlight that our model is disorder-specific, which contributes to the better understanding of $\mathrm{BD}$. We did not include prodromal phases of the disorder because transdiagnostic staging models are probably better suited to the study of at-risk and prodromal phases, while 
disorder-specific models are more appropriate once it has been diagnosed ${ }^{41}$. However, the present results must be interpreted in light of one main limitation. Given that patients had to give signed informed consent prior to inclusion in the study, we were unable to include extremely severe/agitated patients in the model, consequently leading to underrepresentation of such patients in the model. Nevertheless, one of the main strengths of our study is its empirical approach and longitudinal prospective design. This is the first study to follow an entire range of adult patients representing different clinical stages of BD. Previous studies that validated the proposed models focused only on comparison of early vs. late stages, rather than on the full clinical course. Furthermore, our model considers BD a multidimensional disorder requiring five different life domains to classify patients, and the proposed severity classification formula is easy to implement in daily clinical practice.

In conclusion, this proposed staging model conforms to the conceptualization of $\mathrm{BD}$ as a progressive disorder that develops from mild to severe presentations. In this sense, it could help clinicians and researchers to better understand the disorder and, at the same time, to design more accurate and personalized treatment plans.

\section{Acknowledgements}

We thank Sharon Grevet for her English assistance. She was sponsored by research grants. This work was partly supported by the Spanish Ministry of Science and Innovation, Instituto de Salud Carlos III (ref. PI14/02037), The Government of the Principality of Asturias (PCTI-2018-2022 IDI/2018/235), and Fondos Europeos de Desarrollo Regional (FEDER) Lorena de la Fuente-Tomás received a Severo Ochoa grant (PA-17-PF-B16-179) supported by The Government of the Principality of Asturias.

\section{Author details}

${ }^{1}$ Centro de Investigación Biomédica en Red de Salud Mental (CIBERSAM), Madrid, Spain. ${ }^{2}$ Department of Psychiatry, University of Oviedo, Oviedo, Spain. ${ }^{3}$ Instituto de Investigación Sanitaria del Principado de Asturias (ISPA), Oviedo, Spain. ${ }^{4}$ Instituto de Neurociencias del Principado de Asturias (INEUROPA), Oviedo, Spain. ${ }^{5} \mathrm{La}$ Fe University and Polytechnic Hospital and University of Valencia, Valencia, Spain. ${ }^{6}$ Parc Sanitari Sant Joan de Deu, Barcelona, Spain. ${ }^{7}$ University Hospital Mutua Terrassa, University of Barcelona, Barcelona, Spain. ${ }^{8}$ Mental Health Services of Principado de Asturias (SESPA), Oviedo, Spain

\section{Conflict of interest}

The authors declare that they have no conflict of interest.

\section{Publisher's note}

Springer Nature remains neutral with regard to jurisdictional claims in published maps and institutional affiliations.

Received: 4 September 2019 Revised: 18 December 2019 Accepted: 2 January 2020

Published online: 29 January 2020

\section{References}

1. Vieta, E. et al. Early intervention in bipolar disorder. Am. J. Psychiatry $\mathbf{1 7 5}$ 411-426 (2018).

2. Rosa, A. R. et al. Clinical staging in bipolar disorder: focus on cognition and functioning. J. Clin. Psychiatry 75, 450-456 (2014).
3. Grande, I. et al. Staging bipolar disorder: clinical, biochemical, and functional correlates. Acta Psychiatr. Scand. 129, 437-444 (2014).

4. Kauer-Sant'Anna, M. et al. Brain-derived neurotrophic factor and inflammatory markers in patients with early- vs. late-stage bipolar disorder. Int. J. Neuropsychopharmacol. 12, 447-458 (2009).

5. Cao, B. et al. Hippocampal volume and verbal memory performance in latestage bipolar disorder. J. Psychiatr. Res. 73, 102-107 (2016).

6. Siwek, M. et al. Associations of serum cytokine receptor levels with melancholia, staging of illness, depressive and manic phases, and severity of depression in bipolar disorder. Mol. Neurobiol. 54, 5883-5893 (2017).

7. Reininghaus, E. Z. et al. Extracellular matrix proteins matrix metallopeptidase 9 (MMP9) and soluble intercellular adhesion molecule 1 (SICAM-1) and correlations with clinical staging in euthymic bipolar disorder. Bipolar Disord. 18, 155-163 (2016).

8. Mwangi, B. et al. Individualized prediction and clinical staging of bipolar disorders using neuroanatomical biomarkers. Biol. Psychiatry Cogn. Neurosci. Neuroimaging 1, 186-194 (2016).

9. Tatay-Manteiga, A. et al. Clinical staging and serum cytokines in bipolar patients during euthymia. Prog. Neuropsychopharmacol. Biol. Psychiatry 77, 194-201 (2017).

10. Goi, P. D. et al. Pharmacological treatment and staging in bipolar disorder: evidence from clinical practice. Rev. Bras. Psiquiatr. 37, 121-125 (2015).

11. Tatay-Manteiga, A. et al. Subjective neurocognition and quality of life in patients with bipolar disorder and siblings. J. Affect. Disord. 245, 283-288 (2019).

12. Kapczinski, F. et al. Clinical implications of a staging model for bipolar disorders. Expert Rev. Neurother. 9, 957-966 (2009).

13. Berk, M. et al. Setting the stage: from prodrome to treatment resistance in bipolar disorder. Bipolar Disord. 9, 671-678 (2007).

14. Berk, M., Hallam, K. T. \& McGorry, P. D. The potential utility of a staging model as a course specifier: a bipolar disorder perspective. J. Affect. Disord. 100 279-281 (2007).

15. Kapczinski, F. et al. The potential use of biomarkers as an adjunctive tool for staging bipolar disorder. Prog. Neuropsychopharmacol. Biol. Psychiatry 33, 1366-1371 (2009)

16. Duffy, A. Toward a comprehensive clinical staging model for bipolar disorder: integrating the evidence. Can. J. Psychiatry 59, 659-666 (2014).

17. de la Fuente-Tomas, L. et al. Clinical staging in severe mental disorders; bipolar disorder, depression and schizophrenia. Rev. Psiquiatr. Salud Ment. 12, 106-115 (2018).

18. van der Markt, A. et al. Testing a clinical staging model for bipolar disorder using longitudinal life chart data. Bipolar Disord. 21, 228-234 (2019).

19. Cochran, A. L., McInnis, M. G. \& Forger, D. B. Data-driven classification of bipolar I disorder from longitudinal course of mood. Transl. Psychiatry 8, 36 (2016).

20. Cochran, A. L., Shultz, A., Mclnnis, M. G. \& Forger, D. B. Testing frameworks for personalizing bipolar disorder. Transl. Psychiatry 8, 36 (2018).

21. Fuente-Tomas, L. et al. Classification of patients with bipolar disorder using k-means clustering. PLoS ONE 14, e0210314 (2019).

22. American Psychiatric Association. DSM-IV-TR. Diagnostic and Statistical Manual Disorders. (APA, Washington, 2002).

23. Bobes, J. et al. A comparative psychometric study of the Spanish versions with 6, 17, and 21 items of the Hamilton Depression Rating Scale. Med. Clin. 120, 693-700 (2003)

24. Lobo, A. et al. Validation of the Spanish versions of the Montgomery-Asberg depression and Hamilton Anxiety Rating Scales. Med. Clin. 118, 493-499 (2002).

25. Colom, F. et al. Spanish version of a scale for the assessment of mania: validity and reliability of the Young Mania Rating Scale. Med. Clin. 119, 366-371 (2002)

26. Guy, W. Early Clinical Drug Evaluation (ECDEU) Assessment Manual. (National Institute Mental Health, Rockville, 1976).

27. Bobes, J. et al. Propiedades psciométricas del cuestionario de Oviedo de sueño. Psicothema 12, 107-112 (2000).

28. Garcia-Portilla, M. P. et al. Psychometric properties of the Spanish version of the Changes in Sexual Functioning Questionnaire Short-Form (CSFQ-14) in patients with severe mental disorders. J. Sex. Med. 8, 1371-1382 (2011).

29. Gomez-Benito, J. et al. The screen for cognitive impairment in psychiatry: diagnostic-specific standardization in psychiatric ill patients. BMC Psychiatry 13, 127 (2013).

30. American Psychiatric Association. DSM-III-R. Diagnostic and Statistical Manual Disorders. (APA, Washington, 1987). 
31. Rosa, A. R. et al. Validity and reliability of the Functioning Assessment Short Test (FAST) in bipolar disorder. Clin. Pract. Epidemiol. Ment. Health 3, 5 (2007)

32. McHorney, C. A., Ware, J. E. Jr., Lu, J. F. \& Sherbourne, C. D. The MOS 36-item Short-Form Health Survey (SF-36): III. Tests of data quality, scaling assumptions, and reliability across diverse patient groups. Med. Care 32, 40-66 (1994).

33. Nierenberg, A. A. \& DeCecco, L. M. Definitions of antidepressant treatment response, remission, nonresponse, partial response, and other relevant outcomes: a focus on treatment-resistant depression. J. Clin. Psychiatry 62, 5-9 (2001).

34. Kessing, L. V. \& Andersen, P. K. Evidence for clinical progression of unipolar and bipolar disorders. Acta Psychiatr. Scand. 135, 51-64 (2017).

35. da Costa, S. C., Passos, I. C., Lowri, C., Soares, J. C. \& Kapczinski, F. Refractory bipolar disorder and neuroprogression. Prog. Neuropsychopharmacol. Biol. Psychiatry 70, 103-110 (2016).
36. Tatay-Manteiga, A. et al. Staging, neurocognition and social functioning in bipolar disorder. Front. Psychiatry 9, 709 (2018).

37. Czepielewski, L. S. et al. Verbal episodic memory along the course of schizophrenia and bipolar disorder: a new perspective. Eur. Neuropsychopharmacol. 25, 169-175 (2015).

38. Cosci, F. \& Fava, G. A. Staging of mental disorders: systematic review. Psychother. Psychosom. 82, 20-34 (2013).

39. Wesley, M. S., Manjula, M. \& Thirthalli, J. Interepisodic functioning in patients with bipolar disorder in remission. Indian J. Psychol. Med. 40 52-60 (2018).

40. Sole, B. et al. Cognitive impairment in bipolar disorder: treatment and prevention strategies. Int. J. Neuropsychopharmacol. 20, 670-680 (2017).

41. Berk, M. et al. Staging in bipolar disorder: from theoretical framework to clinical utility. World Psychiatry 16, 236-244 (2017). 